\title{
Mass determination of astrometric binaries with Hipparcos
}

\author{
III. New results for 28 systems \\ C. Martin $^{1}$, F. Mignard ${ }^{2}$, W.I. Hartkopf ${ }^{1}$, and H.A. McAlister ${ }^{1}$ \\ 1 Center for High Angular Resolution Astronomy, Georgia State University, Atlanta, Georgia 30303-3083, U.S.A. \\ 2 Observatoire de la Côte d'Azur, CERGA, UMR CNRS 6527, Av. N. Copernic, F-06130 Grasse, France
}

Received April 1; accepted May 20, 1998

\begin{abstract}
This paper is the third of a series devoted to the determination of stellar masses from Hipparcos data. This is a continuation of Martin et al. (1997), who introduced the theory and assessed the performance of the method from simulated data, and of a second paper with the first results for 46 systems, (Martin \& Mignard 1998). The orbit file maintained by the CHARA group and new publications of orbital elements made the processing of 70 additional candidate systems possible, including 28 of the 145 systems already tested in the previous work. Significant results were obtained on 22 systems, with relative accuracy better than $25 \%$ for the masses of 17 binaries. New estimates are also given for 6 systems previously investigated, thanks to reliable values of the magnitude difference from the Hipparcos catalogue (ESA 1997). New orbital elements are proposed for HIP 12623 (12 Persei) from speckle/spectroscopic measurements. Results are discussed for each system, alongside the mass-luminosity relation based on Hipparcos magnitudes and distances.
\end{abstract}

Key words: stars: fundamental parameters — binaries: visual - astrometry

\section{Introduction}

The possibilities to derive stellar masses by a direct method (non model dependent) are not very numerous, and in nearly all cases involve the gravitational attractions in a system formed by two or more stars, (a notable exception to this rule is the binary pulsar PSR $1913+16$, where the individual masses are determined unambiguously from the relativistic periastron shift and the gravitational redshift of the pulsar signal). When the components are close enough to exhibit a fast relative motion and if the

Send offprint requests to: C. Martin, martin@chara.gsu.edu two stars are of comparable brightness, spectroscopic measurements may yield the radial velocity of each component with respect to the centre of mass, and thus the mass ratio of the components. The difficulty in this case is to obtain the total mass from Kepler's third law, since only a function of the semi-major axis and inclination of the relative orbit can be reached by this method. Moreover, the number of objects (double-lined spectroscopic binaries) is not very large. The alternative method, using photographic plates, rests upon the astrometric analysis of the motion of the components (when the separation is larger than $\left.1^{\prime \prime} .5\right)$ or of the photocentre, with respect to nearby stars. When the scale of the relative orbit is known, the previous analysis yields the proper motion, parallax and mass ratio of the system. Whatever the method, its combination with other techniques like speckle interferometry (orbital elements) or visual CCD observations (magnitude difference) is often needed to make the full analysis and derive the individual masses.

The method used in this study has been extensively described in the first paper of the present series (Martin et al. 1997), and is nothing else than a modern version of the astrometric method, with the reservation that in some cases the Hipparcos signal is tied to a point which is not the photocentre (we called it "Hippacentre"). Unlike the situation prevailing with standard astrometric binaries, this fortunate circumstance allows the direct determination of the mass fraction (the independent knowledge of the magnitude difference $\Delta m$ is no longer needed).

This third paper is a continuation of the work presented in Martin \& Mignard (1998). This has been possible essentially through the access to new or previously overlooked orbital data. One of our purposes is to present all the available results, and not only the best ones (it could have been limited to the 10 best results, in good agreement with ground-based determinations), since the anomalous results may question the validity of the orbital elements, or simply reveal the true limitation of the 
Hipparcos sampling. As for Algol in the previous paper, an emphasis is made on a specific system, namely 12 Persei, which has been simultaneously studied by McAlister and his colleagues from a set of ground-based data (this paper, Sect. 5.2). A mass-luminosity relation is also derived from the set of reliable results, including those presented in the previous paper.

For the sake of clarity and conciseness, we will refer in the following to the first two papers of this series as "Paper I" (Martin et al. 1997) and "Paper II" (Martin \& Mignard 1998).

\section{Selection of new candidates and other targets}

\subsection{Origin}

The main result of Paper I was a set of criteria to be met by a binary system, so that one can expect to determine the mass of its components from the Hipparcos astrometric measurements. These criteria are still applicable in the present study (an orbital period $P \leq 30$ years and the semi-major axis $a^{\prime \prime}>$ a few tenths of arcsecond), even if the application to real data has shown that our expectations for pairs with periods larger than 20 years were slightly too optimistic. Two different sources, again largely redundant, were used to select the new candidate systems:

1. The most recent version of the catalog of orbits of visual binary stars compiled by Worley in mid 1997 (not yet published), containing 1403 orbits for 1038 systems.

2. The compilation file of orbits of Scardia (June 1997), also unpublished, containing 1744 orbits for 709 systems.

After elimination of all the objects already studied in our previous paper, and elimination of the systems presenting one or more additional disturbing companions, we are left with 37 systems matching the above criteria in the first source and 5 additional systems in the second one. These 42 new candidates are listed in Table 1.

In addition, we have checked the orbital elements of the 145 systems already processed in Paper II, allowing us in some cases to estimate new masses based on revised orbits, or even get masses for systems where the previous treatment failed.

These 28 systems are listed in Table 2, followed by six systems whose masses were successfully computed in Paper II. For these six binaries flagged "C" in the Hipparcos Catalog (component solution), the current processing is based on the Hipparcos magnitude differences, when they are of comparable or better quality than the ground-based $\Delta m$ that we used before (but the orbital elements remain unchanged). Eventually, the application of our selection rules yields a total of 99 orbits for 76 systems $(42+34)$.
Table 1. HIP numbers of the 42 new preselected systems

\begin{tabular}{rrrrrrrr}
\hline 1242 & 11542 & 28691 & 45383 & 68682 & 86722 & 105200 \\
2548 & 11548 & 30883 & 47250 & 71729 & 95995 & 106255 \\
3951 & 12153 & 31737 & 48029 & 80677 & 96302 & 107288 \\
5064 & 12640 & 34025 & 55016 & 81126 & 97365 & 113031 \\
5317 & 19009 & 36238 & 60129 & 83895 & 101227 & 116164 \\
8903 & 20087 & 38474 & 63510 & 84949 & 102589 & 117761 \\
\hline
\end{tabular}

Table 2. HIP numbers of the 34 reprocessed systems. For the last six systems, masses were already derived in the previous paper

\begin{tabular}{rrrrrrrr}
7918 & 12623 & 22196 & 45571 & 82817 & 94144 & 107788 \\
10535 & 20661 & 29746 & 51147 & 85141 & 98001 & 111528 \\
11452 & 20686 & 33451 & 71094 & 85846 & 103655 & 113445 \\
12421 & 21281 & 39261 & 76852 & 87895 & 104858 & 114576 \\
\hline 2237 & 2762 & 44248 & 84140 & 93574 & 107354 & \\
\hline
\end{tabular}

\subsection{Elimination of objects}

The same rules as in Paper II apply here; no significant results can be obtained for multiple systems if a third (or more) bright components is within $25^{\prime \prime}$ of the pair under consideration. This restriction is directly linked to the Hipparcos observing field. In some cases, when the magnitude of the parasitic source was close to or beyond the sensibility threshold, a solution could however be derived. Among the new candidates, 5 such systems were removed in the present study (18 in Paper II).

\subsection{Description of the systems}

The description of the sample shown in Fig. 1 aims to distinguish between two categories of systems:

1. the so called "Type I" stars for which the mass fraction $B=M_{2} /\left(M_{1}+M_{2}\right)$ can be derived solely from the Hipparcos data (pairs with semi-major axis typically larger than 0 !'3),

2. the "Type II" stars, with a possible determination of the scale factor between the relative and photocentric orbits, namely the difference $\beta-B$ between the intensity and mass fractions.

Although there are five likely candidates of the first kind in this study (exception made of the last 6 systems in Table 2), HIP 7918 was the only object for which a separate computation of the mass fraction was possible. This is primarily due to the limitations caused by the large period characterizing this type of object.

More interesting are the systems with periods smaller than 10 years, making $40 \%$ of the stars selected so far. 
Table 3. The 13 "new" astrometric binaries whose processing yields satisfactory results. The columns give the Hipparcos, ADS and HD identifiers when available, the usual name, the seven orbital elements and their reference

\begin{tabular}{|c|c|c|c|c|c|c|c|c|c|c|c|}
\hline HIP & ADS & $\mathrm{HD}$ & Name $^{1}$ & $a\left({ }^{\prime \prime}\right)$ & $P(\mathrm{yr})$ & $e$ & $i$ & $\Omega$ & $\omega$ & $T$ & ref. $^{2}$ \\
\hline 8903 & - & 11636 & $\beta$ Ari & 0.036 & 0.29 & 0.903 & 44.70 & 79.10 & 209.10 & 1980.098 & Pan90 \\
\hline 12153 & - & 16234 & McA 7, 31 Ari & 0.119 & 1.92 & 0.884 & 101.00 & 171.30 & 256.90 & 1986.830 & Mas97a \\
\hline 20087 & - & 27176 & McA 14, 51 Tau & 0.129 & 11.38 & 0.171 & 125.90 & 170.00 & 160.90 & 1977.830 & Bal89 \\
\hline 55016 & - & 97907 & McA 35, 73 Leo & 0.046 & 8.11 & 0.415 & 49.10 & 283.30 & 156.50 & 1974.015 & Mas97b \\
\hline 60129 & - & 107259 & McA 37,15 Vir & 0.136 & 13.12 & 0.079 & 51.10 & 173.00 & 1.40 & 1963.800 & Har92 \\
\hline 68682 & - & 122742 & - & 0.330 & 9.90 & 0.547 & 93.50 & 252.30 & 189.00 & 1951.960 & $\operatorname{Kam} 87^{3}$ \\
\hline 71729 & - & 129132 & $\mathrm{McA} 40$ & 0.073 & 9.26 & 0.040 & 106.40 & 78.00 & 241.90 & 1984.852 & Bai89 \\
\hline 81126 & - & 149630 & $\sigma$ Her & 0.074 & 7.48 & 0.533 & 108.70 & 13.20 & 175.70 & 1982.560 & Bal89 \\
\hline 83895 & - & 155763 & $\zeta$ Dra & 0.067 & 6.09 & 0.000 & 0.00 & 0.00 & 0.00 & 1980.760 & Zul92 \\
\hline 84949 & - & 157482 & $\mathrm{McA} 47$ & 0.075 & 5.53 & 0.672 & 56.20 & 323.70 & 40.60 & 1986.364 & Sca94 \\
\hline 86722 & - & 161198 & G $170-61$ & 0.174 & 7.00 & 0.936 & 42.80 & 309.20 & 129.60 & 1994.189 & Duq96 \\
\hline 95995 & - & 184467 & McA 56 & 0.084 & 1.35 & 0.370 & 148.10 & 63.70 & 0.00 & 1989.328 & Bai89 \\
\hline 96302 & - & 184759 & $9 \mathrm{Cyg}$ & 0.030 & 4.56 & 0.820 & 114.60 & 29.30 & 45.50 & 1985.560 & Bai89 \\
\hline
\end{tabular}

${ }^{1}$ When available, the more common name of the star, or the discoverer designation.

${ }^{2}$ The three first characters of the author's name followed by the two last digits of the publication's year.

${ }^{3}$ Ground-based measurements yield the size of the photocentric orbit (0". 108$)$. The value 0. ' 33 , which refers to the relative orbit, is just an assumption (see Kamper 1987).

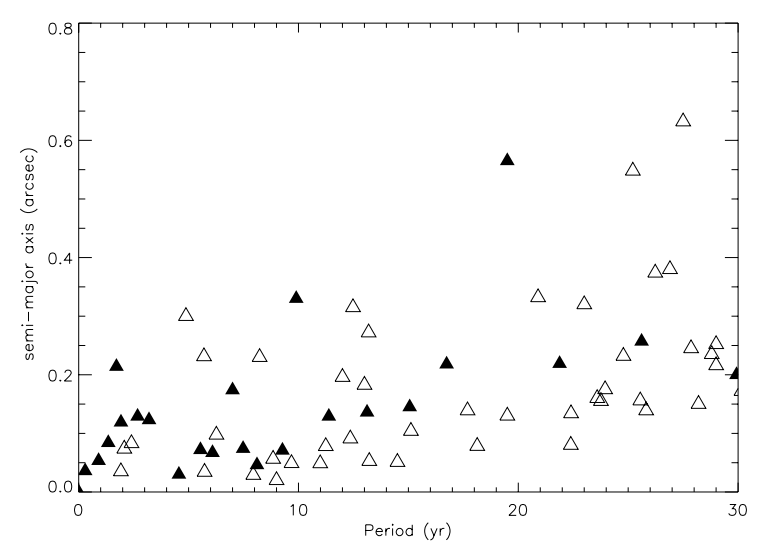

Fig. 1. Distribution of the 70 candidate systems (42 new systems +34 reprocessed, minus the last 6 systems of Table 2 ) in period-semi-major axis space. The filled symbols correspond to the stars whose processing has been successful. Only one orbit per system was considered

Two thirds of the accepted results fall in this group (see Table 6). There are two good reasons accounting for this fact: first, the odds that the orbital motion shows up in the Hipparcos data are more favorable for short periods, and secondly most of these systems have quasi-definitive orbits, allowing a good matching between the set of observations and the model.

\section{Main features of the processing}

The practical implementation of the processing is described in Paper I, Sect. 4.2 and will not be repeated here. The important points are simply summarized below.

The general astrometric processing of the observations carried out by Hipparcos produced for each of the 118300 program stars, the five astrometric parameters representing the position, the parallax and the proper motion. When the program star was single, the previous quantities were associated with the centre of light of the star, that is to say its astrometric direction. In the case of a double star with or without detectable orbital motion, each one-dimensional observation along the scan direction was linked to a point specific to the Hipparcos detection system which was closely related to the photocentre of the pair, but not always identical. The term Hippacentre was coined to convey this idea. For very close pairs, the Hippacentre and the photocentre could not be distinguished by Hipparcos.

For orbital astrometric binaries, the absolute motion of the photocentre $F$ referred to the barycentre $G$, is related to the relative orbit $\rho$ by,

$\mathbf{G F}=(\beta-B) \rho$

where $\beta=\frac{I_{2}}{I_{1}+I_{2}}$ and $B=\frac{M_{2}}{M_{1}+M_{2}}$ are the relative intensity and mass of the secondary component. A more general expression is given in Paper I.

The processing consists of extending the standard Hipparcos astrometric treatment by adding to the usual five astrometric unknowns $\left(l, b, \pi, \mu_{l}\right.$ and $\left.\mu_{b}\right)$ related to 
Table 4. The 9 astrometric binaries unpublished in the previous paper or reprocessed with different orbital elements, followed by 6 revised systems whose masses have already been presented in the previous work

\begin{tabular}{|c|c|c|c|c|c|c|c|c|c|c|c|}
\hline HIP & ADS & $\mathrm{HD}$ & Name $^{1}$ & $a\left({ }^{\prime \prime}\right)$ & $P(\mathrm{yr})$ & $e$ & $i$ & $\Omega$ & $\omega$ & $T$ & ref. $^{2}$ \\
\hline 7918 & - & 10307 & Gl 67 & 0.565 & 19.500 & 0.420 & 104.00 & 32.60 & 100.00 & 1977.600 & Lip83 \\
\hline 12623 & - & 16739 & 12 Per & 0.053 & 0.906 & 0.656 & 126.75 & 230.62 & 89.54 & 1995.148 & this paper \\
\hline 33451 & - & 51825 & I 65 & 0.218 & 16.740 & 0.430 & 35.00 & 128.50 & 241.60 & 1958.850 & Fin63 \\
\hline 45571 & - & 80671 & $128 \mathrm{Car}$ & 0.123 & 3.200 & 0.510 & 123.60 & 156.10 & 294.20 & 1961.730 & Fin64 \\
\hline 71094 & 9301 & 127726 & A 570 & 0.200 & 29.930 & 0.160 & 158.00 & 23.30 & 57.00 & 1983.850 & Hei91 \\
\hline 76852 & 9744 & 140159 & $21 \mathrm{Ser}$ & 0.219 & 21.880 & 0.053 & 83.90 & 70.10 & 74.50 & 1984.880 & Hei94 \\
\hline 82817 & - & 152751 & Kui 75 & 0.214 & 1.717 & 0.049 & 165.70 & 144.50 & 101.00 & 1983.002 & Hei84a \\
\hline 85141 & - & 157498 & Rst 3972 & 0.145 & 15.060 & 0.575 & 20.00 & 43.00 & 46.00 & 1975.700 & Hei97 \\
\hline 98001 & 13125 & 188753 & Но 581 & 0.257 & 25.610 & 0.470 & 31.00 & 61.00 & 217.00 & 1988.060 & Hei90 \\
\hline 2237 & - & 2475 & В 1909 & 0.214 & 11.25 & 0.000 & 69.80 & 119.00 & 0.00 & 1940.880 & Van56 \\
\hline 2762 & 490 & 3196 & 13 Cet & 0.240 & 6.89 & 0.767 & 49.00 & 149.20 & 283.50 & 1973.389 & Har89 \\
\hline 44248 & - & 76943 & $10 \mathrm{Uma}$ & 0.647 & 21.77 & 0.151 & 131.26 & 204.39 & 32.52 & 1993.725 & Har96 \\
\hline 84140 & - & 155876 & Kui 79 & 0.762 & 12.95 & 0.743 & 149.14 & 160.00 & 99.00 & 1991.032 & Har96 \\
\hline 93574 & - & 175986 & Fin 357 & 0.155 & 14.38 & 0.390 & 161.00 & 171.90 & 270.00 & 1976.550 & Hei84b \\
\hline 107354 & 15281 & 206901 & $\kappa$ Peg & 0.236 & 11.60 & 0.313 & 108.04 & 288.85 & 304.17 & 1979.207 & Har89 \\
\hline
\end{tabular}

${ }^{1}$ When available, the more common name of the star, or the discoverer designation.

${ }^{2}$ The three first characters of the author's name followed by the two last digits of the publication's year.

Table 5. Spectral types and Johnson-Morgan's colour indices of the components of 13 binaries. The last column indicates the type of transformation* to be made in order to get the Cousin's $(V-I)$ index (see Sect. 5.2 and Fig. 4 in Paper II)

\begin{tabular}{ccccccc}
\hline HIP & ST $_{1}$ & $\mathrm{ST}_{2}$ & $(V-I)_{1}$ & $(V-I)_{2}$ & Conv. \\
\hline 7918 G1.5 V & M4 V & 0.85 & 3.19 & A & B \\
8903 A5 V & G0 V & 0.22 & 0.81 & A & A \\
12153 F7 V & F8 V & 0.72 & 0.76 & A & A \\
12623 F9 V & F9 V & 0.79 & 0.79 & A & A \\
20087 A8 V & G0 V & 0.31 & 0.81 & A & A \\
33451 F8 IV-V & F8 IV-V & 0.80 & 0.80 & A & A \\
55016 K3 III & F1 V & 1.61 & 0.51 & A & A \\
76852 & B9 V & A1 V & -0.06 & 0.05 & A & A \\
82817 M4.5 V & M4.5 V & 3.33 & 3.33 & B & B \\
84949 G5 IV & F2 V & 1.03 & 0.55 & A & A \\
85141 G0 III & G0 III & 1.09 & 1.09 & A & A \\
86722 K0 V & M3 V & 1.06 & 2.94 & B & B \\
96302 A0 V & F8 III & 0.00 & 1.04 & A & A \\
\hline
\end{tabular}

* Not all the listed systems need this transformation; it depends on the origin of $\Delta m$ (see Tables $7-8$ ).

the centre of mass, the two unknowns $\beta$ and $B$ or simply the combination $\beta-B$ for the closest pairs. The full process goes by successive iterations, starting with reference values $\beta_{\mathrm{r}}$ and $B_{\mathrm{r}}$ of the intensity and mass fractions, until a satisfactory convergence is reached. When several orbits were competing, each of them was tested individ- ually. In each case the reversed orbit was also systematically checked (exchange of the components on the celestial sphere), allowing us to detect an eventual 180 degrees error in the ascending node's position $\Omega$ or in the argument of periastron $\omega$.

In addition to the position and proper motion, the output includes the parallaxes, which can be slightly different from the Hipparcos published values, because our model accounts for the photocentric displacement. In general the effect is very small and within the error bar. The results of this processing are presented in Table 6 .

\section{Results}

The raw results of the processing are presented for 22 systems in Table 6 , in the same layout as in Paper II. Five values of $\beta-B$ have a formal error less than $0.01,3$ are in the range $0.01-0.02$ while the remaining 14 have errors larger than 0.02, a maximum being reached for HIP 55016 (0.084) and HIP 81126 (0.087).

For 16 systems, estimates of $\Delta m$ (and thus of $\beta$ ) are taken from ground-based sources while the Hipparcos data (ESA 1997) were used for the other systems. The transformation into the Hipparcos photometric system was done for the 10 systems with available spectral types for each component (see Table 5). The procedure is described in Paper II, Sect. 5.2. The details of the mass computation will be found in Paper II. 
Table 6. Astrometric binaries processing: raw results for 22 systems. See the orbit's reference in Tables 3-4

\begin{tabular}{|c|c|c|c|c|c|c|c|c|c|c|c|c|}
\hline HIP & Name & orient. & $N_{\mathrm{i}}$ & $N_{\mathrm{p}}$ & $\beta-B$ & $\sigma$ & $B$ & $\sigma$ & $(\beta-B)_{\mathrm{r}}$ & $\sigma$ & $B_{\mathrm{r}}$ & $\sigma$ \\
\hline 7918 & Gl 67 & - & 2 & 7 & -0.145 & 0.049 & 0.147 & 0.050 & -0.230 & 0.014 & 0.231 & 0.014 \\
\hline 8903 & $\beta$ Ari & + & 3 & 6 & -0.332 & 0.047 & - & - & -0.309 & 0.017 & 0.363 & 0.013 \\
\hline 12153 & McA 7 & + & 2 & 6 & -0.008 & 0.014 & - & - & -0.041 & - & 0.495 & - \\
\hline 12623 & 12 Per & + & 2 & 6 & -0.121 & 0.027 & - & - & -0.047 & 0.037 & 0.478 & 0.014 \\
\hline 20087 & McA 14 & + & 3 & 6 & -0.222 & 0.058 & - & - & - & - & - & - \\
\hline 33451 & I 65 & + & 3 & 6 & -0.031 & 0.018 & - & - & - & - & - & - \\
\hline 45571 & $128 \mathrm{Car}$ & + & 3 & 6 & 0.000 & 0.009 & - & - & - & - & - & - \\
\hline 55016 & McA 35 & + & 1 & 6 & -0.400 & 0.084 & - & - & -0.400 & - & 0.465 & - \\
\hline 60129 & McA 37 & - & 6 & 6 & 0.223 & 0.055 & - & - & 0.051 & 0.102 & 0.273 & 0.022 \\
\hline 68682 & - & - & 3 & 6 & -0.312 & 0.007 & - & - & -0.301 & - & 0.320 & - \\
\hline 71094 & A 570 & + & 3 & 6 & 0.013 & 0.008 & - & - & -0.047 & 0.036 & 0.440 & 0.030 \\
\hline 71729 & McA 40 & + & 3 & 6 & -0.109 & 0.065 & - & - & 0.029 & - & 0.358 & - \\
\hline 76852 & $21 \mathrm{Ser}$ & + & 11 & 6 & -0.028 & 0.033 & - & - & - & - & - & - \\
\hline 81126 & $\sigma$ Her & - & 4 & 6 & -0.144 & 0.087 & - & - & - & - & - & - \\
\hline 82817 & Kui 75 & + & 4 & 6 & -0.149 & 0.005 & - & - & -0.019 & 0.097 & 0.500 & 0.084 \\
\hline 83895 & $\zeta$ Dra & - & 3 & 6 & -0.103 & 0.023 & - & - & - & - & - & - \\
\hline 84949 & McA 47 & + & 3 & 6 & -0.208 & 0.012 & - & - & - & - & 0.587 & 0.015 \\
\hline 85141 & Rst 3972 & + & 3 & 6 & -0.040 & 0.042 & - & - & - & - & - & - \\
\hline 86722 & G $170-61$ & + & 10 & 6 & -0.238 & 0.032 & - & - & -0.267 & - & 0.291 & - \\
\hline 95995 & McA 56 & + & 3 & 6 & -0.055 & 0.009 & - & - & - & - & - & - \\
\hline 96302 & 9 Cyg & + & 2 & 6 & 0.034 & 0.043 & - & - & - & - & - & - \\
\hline 98001 & Но 581 & + & 6 & 6 & -0.113 & 0.047 & - & - & -0.310 & 0.030 & 0.656 & 0.020 \\
\hline
\end{tabular}

orient.: The orientation is that of the published orbit $(+)$ or has been reversed after examination of the residuals $(-)$.

$N_{\mathrm{i}}, N_{\mathrm{p}}$ : Number of iterations and number of unknowns in the model.

$\beta-B, B$ : the two "physical" solutions of the reduction followed by the corresponding standard errors.

$(\beta-B)_{\mathrm{r}}, B_{\mathrm{r}}$ : reference values used to start the algorithm (see Table 9 ).

\section{Comments on individual systems}

\subsection{General cases}

- HIP 7918 (Gl 67): A close astrometric binary comprised of a solar-type star and a cool low-mass companion 1000 times fainter. In this sample of 70 candidates, this is the only system with a direct determination of the fractional mass (a consequence of its large separation, see Paper I). The companion was resolved for the first time by Henry et al. (1992), using infrared speckle imaging techniques. With the same orbital elements, Kepler's third law and the Hipparcos parallax yield a total mass about $30 \%$ smaller than Henry's value. The most surprising discrepancy lies with the fractional mass $B$ of the companion, significantly smaller in this study. The combination of both effects yields a secondary's mass about half the value derived by Henry, although affected by a large relative error. Our result may be not very reliable, as suggested by the large correlations found between the unknowns.

- HIP 8903 ( $\beta$ Ari): Famous double-lined spectroscopic binary with an unusually large orbital eccentricity. The new Hipparcos parallax value, slightly larger than the one determined by Pan et al. (1990), leads to smaller indi- vidual masses, but still consistent with Pan's estimates of $2.34 \pm 0.10$ and $1.34 \pm 0.07 M_{\odot}$.

- HIP 12153 (31 Ari): This object was first detected as double by lunar occultation (Africano et al. 1978), then studied with speckle interferometry and spectroscopy. A preliminary orbit has been computed very recently from speckle data (Mason 1997a), in good agreement with the Hipparcos observations. It provides an accurate estimate of $\beta-B$. The true value of the parallax remains puzzling however. Balega's dynamical value of 21 mas (Balega \& Balega 1988) is not consistent with Hipparcos (28.15 mas), still too small to yield realistic masses for this system (see the position of HIP 12153 in the mass-luminosity diagram, Fig. 2). If the latter value is confirmed, a revision of both the orbital period and the size of the relative orbit will be needed. The value of $\Delta m$ taken here is the average of two estimates (Africano et al. 1978), one in the red (0.3), the other in the blue (0.1).

- HIP 20087 (51 Tau): Single-lined spectroscopic binary, whose probable large magnitude difference is not yet accurately known. Considering the dynamical parallax, Baize (1989) suggested a total mass of $3.4 M_{\odot}$ and accounted for the large mass of the secondary (about $1.6 M_{\odot}$ ) by assuming that the star is an evolved subgiant. The new 
Table 7. Masses of 22 systems. See comments at the end of the table

\begin{tabular}{|c|c|c|c|c|c|c|c|c|c|c|c|c|c|c|}
\hline HIP & Corr. ${ }^{(1)}$ & $\begin{array}{c}\pi \\
\operatorname{mas}\end{array}$ & $\begin{array}{c}\sigma_{\pi} \\
\text { mas }\end{array}$ & $\begin{array}{c}\Delta \pi^{(2)} \\
\operatorname{mas}\end{array}$ & $\begin{array}{l}\sigma_{\Delta \pi} \\
\text { mas }\end{array}$ & $\Delta m^{(3)}$ & $\sigma^{(4)}$ & $\begin{array}{c}M \\
M_{\odot}\end{array}$ & $\begin{array}{c}\sigma \\
M_{\odot}\end{array}$ & $\begin{array}{l}M_{1} \\
M_{\odot}\end{array}$ & $\begin{array}{c}\sigma \\
M_{\odot}\end{array}$ & $\begin{array}{l}M_{2} \\
M_{\odot}\end{array}$ & $\begin{array}{c}\sigma \\
M_{\odot}\end{array}$ & $N^{(5)}$ \\
\hline 7918 & yes & 79.86 & 0.91 & 0.77 & 1.23 & 7.451 & 0.50 & 0.931 & 0.178 & 0.795 & 0.159 & 0.136 & 0.053 & 3 \\
\hline 8903 & yes & 54.55 & 0.99 & -0.19 & 1.24 & 3.180 & 0.22 & 3.348 & 0.201 & 2.067 & 0.203 & 1.281 & 0.178 & 2 \\
\hline 12153 & yes & 28.15 & 1.09 & -0.25 & 1.39 & 0.205 & 0.10 & 20.408 & 3.509 & 11.001 & 1.969 & 9.407 & 1.707 & 3 \\
\hline 12623 & yes & 41.94 & 1.56 & 1.42 & 2.00 & 0.300 & 0.15 & 2.514 & 0.289 & 1.125 & 0.169 & 1.389 & 0.193 & 3 \\
\hline 20087 & yes & 18.29 & 0.97 & 0.04 & 1.27 & 2.066 & 0.40 & 2.709 & 0.437 & 1.756 & 0.343 & 0.953 & 0.247 & 3 \\
\hline 33451 & - & 23.44 & 0.62 & 0.29 & 0.84 & 0.410 & 0.25 & 2.871 & 0.228 & 1.614 & 0.211 & 1.256 & 0.195 & 1 \\
\hline 45571 & no & 29.17 & 0.57 & -0.66 & 0.83 & 0.000 & 0.15 & 7.322 & 0.429 & 3.661 & 0.338 & 3.661 & 0.338 & 1 \\
\hline 55016 & yes & 6.72 & 0.94 & -0.10 & 1.24 & 2.830 & 0.40 & 4.938 & 2.102 & 2.624 & 1.197 & 2.315 & 1.075 & 3 \\
\hline 60129 & no & 12.98 & 0.90 & -0.08 & 1.23 & 0.800 & 0.50 & 6.682 & 2.250 & 6.009 & 2.164 & 0.673 & 0.800 & 3 \\
\hline 68682 & no & 60.50 & 0.97 & 0.26 & 1.24 & 4.300 & 0.50 & 1.657 & 0.080 & 1.109 & 0.057 & 0.548 & 0.033 & 2 \\
\hline 71094 & - & 14.30 & 1.02 & 0.27 & 1.37 & 0.470 & 0.09 & 3.054 & 0.654 & 1.892 & 0.410 & 1.162 & 0.257 & 1 \\
\hline 71729 & no & 8.81 & 0.75 & -0.66 & 1.03 & 1.000 & 0.20 & 6.633 & 1.694 & 3.344 & 1.000 & 3.289 & 0.988 & 1 \\
\hline 76852 & - & 17.66 & 0.93 & 0.69 & 1.21 & 0.153 & 0.06 & 3.984 & 0.629 & 1.999 & 0.346 & 1.984 & 0.344 & 1 \\
\hline 81126 & no & 11.69 & 0.70 & 0.90 & 0.93 & 1.600 & 0.30 & 4.534 & 0.842 & 3.036 & 0.714 & 1.498 & 0.519 & 3 \\
\hline 82817 & - & 155.42 & 1.85 & -18.81 & 4.32 & 0.080 & 0.21 & 0.885 & 0.032 & 0.327 & 0.045 & 0.558 & 0.047 & 1 \\
\hline 83895 & no & 9.46 & 0.56 & -0.14 & 0.73 & 1.040 & 0.20 & 9.585 & 1.702 & 5.940 & 1.134 & 3.645 & 0.770 & 1 \\
\hline 84949 & yes & 15.41 & 0.63 & -0.12 & 1.32 & 0.055 & 0.20 & 3.774 & 0.469 & 1.150 & 0.229 & 2.624 & 0.372 & 3 \\
\hline 85141 & yes & 15.60 & 1.30 & 0.09 & 1.75 & 0.200 & 0.20 & 3.541 & 0.885 & 1.791 & 0.499 & 1.749 & 0.489 & 1 \\
\hline 86722 & yes & 43.80 & 1.29 & 1.35 & 1.62 & 3.958 & 0.50 & 1.279 & 0.217 & 0.942 & 0.166 & 0.337 & 0.072 & 3 \\
\hline 95995 & - & 58.01 & 0.69 & -1.83 & 0.94 & 1.390 & 0.63 & 1.673 & 0.060 & 1.217 & 0.172 & 0.456 & 0.167 & 1 \\
\hline 96302 & yes & 6.41 & 0.72 & 0.35 & 0.92 & 0.645 & 0.20 & 4.930 & 1.661 & 3.344 & 1.165 & 1.586 & 0.612 & 1 \\
\hline 98001 & - & 22.06 & 0.78 & -0.25 & 1.10 & 0.690 & 0.11 & 2.411 & 0.256 & 1.304 & 0.187 & 1.107 & 0.172 & 1 \\
\hline
\end{tabular}

\footnotetext{
${ }^{1}$ Correction: "yes" indicates that the $\Delta m$ estimate has been brought into the Hipparcos photometric system, via the spectral types of the components (see Sect. 5.2). "no" means that this conversion could not be done due to the lack of individual spectral types, and "-" means that $\Delta m$ is taken from the Hipparcos catalogue and thus does not need any conversion.

${ }^{2}$ Difference between the parallax derived from this processing and the Hipparcos catalogue's value (ESA 1997), followed by its standard deviation. Most of the differences are not significant, except for HIP $82817=$ Kui75, where the catalogue's value is probably wrong.

${ }^{3}$ It is the Hipparcos estimate if "_" stands in the second column, a ground-based one otherwise.

${ }^{4}$ When unknown, $\sigma$ is taken equal to 0.15 .

${ }^{5}$ Number of available terms for the computation of the standard deviation of the total mass $M . N=3$ if $\sigma_{\mathrm{a}}, \sigma_{\pi}$ and $\sigma_{\mathrm{P}}$ are known. $N=2$ if $\sigma_{\mathrm{P}}$ is unknown. $N=1$ if both $\sigma_{\mathrm{a}}$ and $\sigma_{\mathrm{P}}$ are ignored. In the latter case, $\sigma(M)$ is underestimated.
}

Table 8. Masses for 6 systems already studied in the previous paper, reprocessed with the Hipparcos magnitude difference estimate. See comments at the end of the previous table

\begin{tabular}{|c|c|c|c|c|c|c|c|c|c|c|c|c|c|c|}
\hline HIP & Corr. ${ }^{(1)}$ & $\begin{array}{c}\pi \\
\text { mas }\end{array}$ & $\begin{array}{c}\sigma_{\pi} \\
\text { mas }\end{array}$ & $\begin{array}{c}\Delta \pi^{(2)} \\
\operatorname{mas}\end{array}$ & $\begin{array}{l}\sigma_{\Delta \pi} \\
\text { mas }\end{array}$ & $\Delta m^{(3)}$ & $\sigma^{(4)}$ & $\begin{array}{c}M \\
M_{\odot}\end{array}$ & $\begin{array}{c}\sigma \\
M_{\odot}\end{array}$ & $\begin{array}{l}M_{1} \\
M_{\odot}\end{array}$ & $\begin{array}{c}\sigma \\
M_{\odot}\end{array}$ & $\begin{array}{l}M_{2} \\
M_{\odot}\end{array}$ & $\begin{array}{c}\sigma \\
M_{\odot}\end{array}$ & $N^{(5)}$ \\
\hline 2237 & - & 30.24 & 0.98 & -0.77 & 1.31 & 0.17 & 0.12 & 2.800 & 0.272 & 1.563 & 0.177 & 1.238 & 0.151 & 1 \\
\hline 2762 & - & 46.74 & 0.97 & -0.77 & 1.50 & 1.29 & 0.07 & 2.852 & 0.425 & 1.712 & 0.296 & 1.140 & 0.226 & 3 \\
\hline 44248 & - & 61.61 & 1.13 & 0.75 & 1.72 & 2.30 & 0.04 & 2.445 & 0.135 & 1.525 & 0.104 & 0.920 & 0.080 & 3 \\
\hline 84140 & - & 152.20 & 1.82 & -5.97 & 3.73 & 0.23 & 0.17 & 0.748 & 0.027 & 0.379 & 0.035 & 0.369 & 0.035 & 3 \\
\hline 93574 & - & 17.20 & 0.61 & -0.45 & 0.99 & 0.38 & 0.21 & 3.539 & 0.377 & 1.892 & 0.265 & 1.647 & 0.246 & 1 \\
\hline 107354 & - & 28.63 & 0.92 & 0.29 & 1.27 & 0.10 & 0.11 & 4.163 & 0.411 & 1.561 & 0.197 & 2.602 & 0.284 & 3 \\
\hline
\end{tabular}


parallax and mass fraction estimates confirm the mass of the primary (about $1.8 M_{\odot}$ ) but yield a companion's mass more typical of a main sequence dwarf with a G0 spectral type $\left(\approx 1 M_{\odot}\right)$.

- HIP 33451 (I 65): Visual/speckle binary mostly observed by visual techniques during the last century. The orbit is flagged "definitive" in the catalog of Worley \& Heintz (1983). Unfortunately we did not find any information concerning the masses of the components. Our masses and absolute magnitudes agree nicely with the empirical massluminosity relation.

- HIP 45571 (128 Car): First detected as double in 1960, this system is in fact triple, the $\mathrm{C}$ component with a magnitude 12.2 lies at $18^{\prime \prime}$ from the close AB binary. At this distance from the central pair, the attenuation effect caused by the Hipparcos dissector tube makes the $\mathrm{C}$ component nearly invisible, and thus too faint to disturb the signal of $\mathrm{AB}$. The magnitude difference derived from Hipparcos is very poor $(1.37 \pm 0.87)$ and we have used the value given by Worley. This pair is one of the 6 stars of this sample with a standard deviation of $\beta-B$ smaller than 0.01 . The two masses are found nearly equal which suggests either a pair of two G7 giant stars, or a pair of A0 dwarfs. This is not compatible with the absolute magnitudes, however (see the mass luminosity relation, Fig. 2). The orbital elements are indeed still preliminary and need to be confirmed.

- HIP 55016 (73 Leo): Speckle and spectroscopic binary, the very first star to have a photoelectric velocity published (see Griffin 1990). As it is mentioned by Mason (1997b), the exact determination of spectral types and $\Delta m$ are now no clearer than they were in 1990 , so the mass fraction proposed in this paper is not very reliable (we have taken $\Delta m \approx 2.9$, which is probably too large). Unfortunately the high relative error of the parallax does not improve the situation and yields an inaccurate total mass. This system will probably remain problematic for another few years, since a recent attempt to study it with adaptive optics has failed.

- HIP 60129 (McA 37): $\eta$ Virginis is a triple system formed by a close spectroscopic pair (undetectable by Hipparcos) and a more distant speckle companion. Again, the large relative errors of the parallax and semi-major axis prevent a good determination of the total mass. The even worst quality of the individual masses is caused by the uncertain value of $\Delta m$, as derived by speckle interferometry (Hartkopf et al. 1992). The masses and the mass ratio listed as references in Table 9 are based on arbitrary assumptions and must be considered with caution. Due to its long orbital period, $\eta$ Vir clearly deserves further investigations, both with adaptive optics $(\Delta m)$ and speckle (orbital elements).
- HIP 68682 (HR 5273): Astrometric-spectroscopic binary, recently studied by Kamper (1987). This is one of the 3 best results of the present research (mass uncertainties at about $5 \%$ ), but some restrictions must be mentioned. The secondary component is actually unseen and the $\Delta m$ proposed by Kamper and used in this study is uncertain. This is not a serious problem however, since the value of the fractional intensity $\beta$ is anyway close to zero and the calculation of the fractional mass $B$ is not very sensitive to a possible error of $\Delta m$. More important is the consequence of the semi-major axis of the relative orbit, estimated at about 0 ". 33 by Kamper, a value based on uncertain assumptions (see the two last paragraphs of the previously mentioned paper). The adoption of a different value would of course change the estimate of $B$ and of the total mass $M$. Taking $a=0^{\prime \prime} \cdot 33$ yields a mass ratio perfectly consistent with Kamper's value, but our mass estimates are slightly larger, a consequence of the Hipparcos parallax. The periastron argument $\omega$ should be rotated by 180 degrees.

- HIP 71094 (A 570): Variable speckle binary, one of the best results regarding $\beta-B$ despite the long period. The fairly small parallax is consistent with the dynamical estimate of $15 \pm 5$ mas (Heintz 1991). The lack of individual spectral types for this system makes the validation of the masses difficult. The location of the two components in the mass-luminosity diagram is reasonable.

- HIP 71729 (McA 40): Single-lined spectroscopic and speckle triple system formed by a very short-period $(\approx 100$ days) pair and a more distant speckle companion. The closest companion was unresolved by Hipparcos and the primary is in fact composite. The new parallax yields a total mass about $30 \%$ larger than the previous estimate by Barlow \& Scarfe (1991). The mass ratio also differs, so that the total mass of the primary component $(\mathrm{Aa}+\mathrm{Ab})$ is conserved, while the secondary mass appears to be much larger, with fairly large underestimated relative errors. We are not very confident in these new results. The magnitude difference and the spectral types also need more checks.

- HIP 76852 (21 Ser): Astrometric/speckle binary. No mass estimates have been found in the literature, but the spectral types B9V and A1V are not compatible with the masses derived here (they suggest masses close to $3 M_{\odot}$ instead of $2 M_{\odot}$ ). The new parallax compares quite well with previous estimates and is probably not suspect. Thus, either the orbital elements (there is still a doubt regarding the period) or the spectral types may be wrong. A couple of $\mathrm{A} 4 \mathrm{~V}$ stars would fit better.

- HIP 81126 ( $\sigma$ Her): Speckle binary star containing an object suspected to be a $\beta$ Pictoris-like star, due to its large colour excess in the infrared. The mass excess mentioned by Baize (1989), based on a dynamical parallax close to 10 mas (Balega \& Balega 1988) is not confirmed here, due to the new parallax estimate. The value of $\Delta m$ is 
still not very clear, since Balega proposed $1.6 \pm 0.3$ instead of 3.5 , as previously assumed.

- HIP 82817 (Kui 75): Famous UV emitting flare star and visual/speckle binary. This is the only system in the present sample for which the new parallax produced by the specific processing for short-period binaries is significantly different (about 12\% smaller) from the catalogue's value (ESA 1997). This new estimate (155.42 \pm 1.85 mas) agrees well with the most recent ground-based trigonometric determination: $152 \pm 4$ (Jenkins 1963) and is much more accurate than the Hipparcos value. The latter determination was in fact quite uncertain because no orbital model was used in the data reduction, and there is little doubt that the new value is closer to reality. This situation is very akin to that of Algol.

- HIP 83895 ( $\zeta$ Dra): The most recent paper concerning this object (Olević et al. 1997) supports a significant change in the orbital elements previously computed by Zulević (1992). The new elements are still considered as preliminary, however, and indeed do not improve the results already obtained with the former orbit. Unfortunately, masses and parallax of the pair were not calculated because of the lack of magnitudes and spectra, as mentioned by Olević (1997). It is thus difficult to assess the quality of the present results, based on Zulević's orbit (1992) and differential photometry (1993). The total mass derived here $\left(\approx 10 M_{\odot}\right)$ suggests a pair of giants.

- HIP 84949 (McA 47, HR 6469): Speckle/spectroscopic triple system consisting of a close eclipsing pair containing a F2V star, orbitting a more distant G5IV variable component. This very interesting system was the subject of three papers published at the same time (Wasson et al.; Van Hamme et al.; Scarfe et al. 1994) in the same journal. We have used the more recent orbital elements proposed by Scarfe et al. (1994) together with $\Delta m=0$ as suggested by Baize (1991), although this must be used with care because of the photometric peculiarities of the system. The evolved G5 star is referred to as the primary and has a period of variability of about 83 days in $\mathrm{V}$, while the secondary is taken as the brighter star of the close eclipsing pair, whose period is 2.23 days (Van Hamme 1994). The duplicity of the second component could not be seen by Hipparcos, and the double variability phenomenon (eclipse + spotted variable) entails reasonably small change of the magnitude $(<0.09 \mathrm{mag})$, so the set of Hipparcos observations may be considered as photometrically homogeneous, at least for the purpose of this study. We obtain a mass of $1.15 M_{\odot}$ for the evolved primary and a total mass of $2.63 M_{\odot}$ for the eclipsing pair, with formal errors of $20 \%$ and $14 \%$ respectively. While the mass of the secondary is perfectly consistent with Scarfe's determination, it is not true for the primary $\left(1.86 \pm 0.09 M_{\odot}\right)$. This can be traced to small discrepancies in the estimates of the parallax and the fractional mass. Combining Scarfe's fractional mass
$B=0.587$ with our $\beta-B$ yields an estimate of $\beta$ and thus of the approximative magnitude difference between the evolved primary and the close eclipsing pair, $\Delta m \approx 0.54$ (in the Hipparcos band), half a magnitude larger than Baize's. This assumption leads to individual masses of $1.56 \pm 0.31 M_{\odot}$ and $2.21 \pm 0.31 M_{\odot}$. The composite secondary has been excluded from the mass-luminosity diagram (Sect. 6.2) and thus does not contribute to the fit.

- HIP 85141 (Rst 3972): Visual/speckle binary formed by two G0 giant stars. For many years the orbital period was assumed to be close to 30 years, until Hartkopf et al. (1996) set forth a half-period alternative, which fits better the recent measurements. We used these orbital elements in Paper II to derive masses of $1.51 M_{\odot}$ and $2.94 M_{\odot}$ with formal errors at the level of $30 \%$. These elements are now superseded by the new orbit computed by Heintz (1997), in better agreement with the whole set of obervations. We find this time two identical masses of about $1.8 M_{\odot}$, with errors at the same level. Values of $\beta-B$ and $\Delta m$ agree well with the fact that both components have indentical spectral types, but the total mass is slightly too small for two G0III stars. If we consider the smallest possible parallax allowed by the error bar $(\approx 14.3$ mas $)$, we find a total mass of $4.6 M_{\odot}$, closer to the expected value.

- HIP 86722 (Gl 692.1): Single-lined spectroscopic and speckle binary, recently studied by Duquennoy using the CORAVEL and RVM radial-velocity spectrometers and near IR speckle data (Duquennoy et al. 1996). All the values derived here (masses and absolute magnitudes) agree perfectly with Duquennoy's assumptions, except for the mass of the primary component, which we found to be $20 \%$ larger. These new results are the most accurate to date and must replace any previous determination.

- HIP 95995 (McA 56): This K star of the solar neighbourhood is a speckle-spectroscopic binary containing a low mass companion, mainly noticed for its high proper motion. Very precise total mass and $\beta-B$ have been determined in this study, but the quality of $\Delta m$ is still too poor and causes the estimates of the individual masses to be rather uncertain. Better differential photometry is needed.

- HIP 96302 (9 Cyg): Single-lined spectroscopic binary presenting one of the smallest separations of this set. As with all the most distant stars, the masses are not very reliable. The $\Delta m$ estimate of Baize (1989) does not agree with the value (which we have adopted) found in the Worley catalogue. Moreover, the period is not accurately known.

- HIP 98001 (Ho 581): Visual binary star containing a spectroscopic system (the A component is associated with a low mass spectroscopic companion, see Griffin 1997). The parallax and total mass derived here are 
Table 9. Reference values of component's masses and physical ratios

\begin{tabular}{|c|c|c|c|c|c|c|c|c|c|c|}
\hline HIP & $\begin{array}{l}M_{1} \\
M_{\odot}\end{array}$ & $\begin{array}{c}\sigma \\
M_{\odot}\end{array}$ & $\begin{array}{l}M_{2} \\
M_{\odot}\end{array}$ & $\begin{array}{c}\sigma \\
M_{\odot}\end{array}$ & $B$ & $\sigma$ & ref. $^{1}$ & $\beta$ & $\sigma$ & ref. $^{1}$ \\
\hline 7918 & 0.93 & 0.23 & 0.28 & 0.07 & 0.231 & 0.014 & Hen93 & 0.001 & $<0.001$ & Hen93 \\
\hline 8903 & 2.34 & 0.10 & 1.34 & 0.07 & 0.363 & 0.013 & Pan90 & 0.054 & 0.010 & Pan90 \\
\hline 12153 & $1.20 ?$ & - & $1.20 ?$ & - & 0.500 & - & Afr78 & 0.454 & 0.023 & Afr78 \\
\hline 12623 & 1.28 & 0.05 & 1.17 & 0.05 & 0.478 & 0.014 & This paper & 0.431 & 0.034 & Col35 \\
\hline 20087 & 1.80 & - & 1.60 & - & 0.470 & - & Bai89 & 0.137 & 0.043 & Bai89 \\
\hline 33451 & - & - & - & - & - & - & - & 0.407 & 0.055 & ESA97 \\
\hline 45571 & - & - & - & - & - & - & - & 0.500 & 0.034 & Wor96 \\
\hline 55016 & $2.07 ?$ & - & $1.80 ?$ & - & 0.465 & - & Mas97b & 0.065 & 0.022 & Mas97b \\
\hline 60129 & 4.29 & 0.40 & 1.61 & 0.10 & 0.273 & 0.022 & Har92 & 0.324 & 0.100 & Har92 \\
\hline 68682 & 0.85 & - & 0.40 & - & 0.320 & - & Kam87 & 0.019 & 0.008 & Kam87 \\
\hline 71094 & - & - & - & - & 0.440 & 0.030 & Hei91 & 0.393 & 0.020 & ESA97 \\
\hline 71729 & 3.26 & - & 1.82 & - & - & - & Bar91 & 0.387 & 0.045 & Bar91 \\
\hline 76852 & - & - & - & - & - & - & - & 0.470 & 0.014 & ESA97 \\
\hline 81126 & - & - & - & - & - & - & - & 0.186 & 0.042 & Bal88 \\
\hline 82817 & 0.42 & 0.10 & 0.42 & 0.10 & 0.500 & 0.084 & Mal93 & 0.481 & 0.048 & ESA97 \\
\hline 83895 & - & - & - & - & - & - & - & 0.277 & 0.037 & Zul93 \\
\hline 84949 & 1.86 & 0.09 & 2.64 & 0.10 & 0.587 & 0.015 & Sca94 & 0.500 & 0.046 & Bai91 \\
\hline 85141 & - & - & - & - & - & - & - & 0.454 & 0.046 & Hei97 \\
\hline 86722 & 0.78 & - & 0.32 & - & 0.291 & - & Duq96 & 0.024 & 0.011 & Duq96 \\
\hline 94144 & - & - & - & - & - & - & - & 0.452 & 0.023 & Bai50 \\
\hline 95995 & - & - & - & - & - & - & - & 0.217 & 0.098 & ESA97 \\
\hline 96302 & - & - & - & - & - & - & - & 0.387 & 0.044 & Wor96 \\
\hline 98001 & - & - & - & - & 0.656 & 0.020 & Hei90 & 0.346 & 0.023 & ESA97 \\
\hline
\end{tabular}

1 The three first characters of the author's name followed by the two last digits of the publication's year.

compatible with the determination of Heintz (1990), but the very large mass fraction $(B=0.656)$ is not confirmed here. We find a $B=0.459 \pm 0.052$, yielding a primary component slightly more massive than the secondary. The positions of both stars in the empirical mass-luminosity diagram look satisfactory.

\subsection{A special attention for 12 Per (HIP 12623)}

The system was identified by McAlister (1976) as a likely candidate for resolution by speckle interferometry, and, indeed, the two components had been resolved by C.R. Lynds in an unpublished, exploratory speckle program at the Kitt Peak 4-meter telescope in 1973. Routine speckle measurements of the system were begun in 1975, and McAlister (1976) published an orbital solution based upon five speckle measurements and the spectroscopic elements of Colacevich (1941). Some 40 speckle measures are now available for analysis. We have calculated new orbital elements for this system, presented in Table 11. They do not differ strongly from the previous estimations, see for example McAlister (1978) although the formal errors are significantly reduced. The $\Delta m$ estimate of 0.30 is originally from Colacevich (1935), and no better value has been proposed since then. A confirmation would be especially helpful for a mass determination free of bias. We have studied this system in two different ways:

1. The specific processing from Hipparcos data and a ground-based orbit (like all the other stars of this paper),

2. A combination of available spectroscopic and speckle interferometric data for a three-dimensional orbit solution.

Because of the quantity and quality of available data, the second method yields better results. The available radial velocity data span 97 orbital revolutions of 12 Persei while the speckle measurements cover 25 orbits. The relevant data for 12 Persei have been analyzed thanks to the reduction softwares routinely used for CHARA binary star orbit studies, namely a least-squares "grid search" algorithm for the speckle observations and a program developed by Tokovinin for combining radial velocities and speckle astrometry.

In a first step, the orbital elements for 12 Per given in Table 11 were determined from the entire dataset composed of speckle measurements from the CHARA Catalogue of Speckle Interferometric Measurements of Binary Stars (maintained by W.I. Hartkopf and available on-line) and of the radial velocities of Colacevich (1935, 
Table 10. Comparison of parameters for 12 Persei

\begin{tabular}{crlll}
\hline \multicolumn{1}{c}{ origin } & $\pi(\operatorname{mas})$ & $M\left(M_{\odot}\right)$ & $M_{1}\left(M_{\odot}\right)$ & $M_{2}\left(M_{\odot}\right)$ \\
\hline Ground-based & 42.6 & 2.446 & 1.276 & 1.170 \\
& 1.0 & 0.067 & 0.046 & 0.049 \\
Hipparcos & 41.9 & 2.51 & 1.13 & 1.39 \\
& 1.6 & 0.29 & 0.17 & 0.19 \\
\hline
\end{tabular}

Table 11. New speckle orbital elements of 12 Per

\begin{tabular}{rrrrrrr}
\hline$a\left({ }^{\prime \prime}\right)$ & $P(\mathrm{~d})$ & $e$ & $i$ & $\Omega$ & $\omega$ & \multicolumn{1}{c}{$T$} \\
\hline 0.0534 & 330.957 & 0.656 & 126.75 & 230.62 & 89.54 & 1995.1475 \\
.0005 & .013 & .005 & .62 & .82 & .41 & .0023 \\
\hline
\end{tabular}

1941) and Duquennoy \& Mayor (1991). The orbital period from this solution was then fixed and the remaining orbital elements were determined by combining the speckle data with the Duquennoy and Mayor spectroscopic data, leaving out the old measurements of Colacevich. Independent orbits from these two datasets show excellent agreement between common elements.

The combination of the two data types also permits the determination of an orbital parallax of $42.6 \pm 1.0$ mas which is in excellent agreement with the Hipparcos parallax of $41.9 \pm 1.6$ mas corrected for the orbital motion of the binary.

While we adopt the masses derived from the combined speckle/spectroscopic analysis, we note that masses can also be derived by applying the Hipparcos parallax to the ground-based orbit, i.e. option number 1 above. Table 10 shows the different values obtained by each method.

The discrepancy observed for the individual masses probably arises from the fact that the value of $\Delta m$ used to retrieve the fractional mass $B$ in the first method is uncertain. Assuming the values $B=0.478$ (groundbased) and $\beta-B=-0.121$ (Hipparcos) are correct, we find $\Delta m=0.64 \pm 0.10$, which is not unrealistic. With the Hipparcos parallax, this would produce the following masses: $M_{1}=1.31 \pm 0.16$ and $M_{2}=1.20 \pm 0.13$. An accurate determination of $\Delta m$ would result in truly definitive masses for this system, and we note that ground-based, long-baseline optical interferometers offer this potential.

\subsection{The 6 revised systems}

The results presented in Table 8 for 6 revised systems of Paper II are simply an update of the individual masses and their errors, coming from a more reliable $\Delta m$ estimate. Thus, the parallaxes and total masses are unchanged. The modifications are briefly discussed below.
- HIP 2237 (B 1909): The slight increase of $\Delta m$ makes the mass of the secondary closer to the primary's one.

- HIP 2762 (Kui 7): $\Delta m$ is unchanged but known with a better precision, resulting in more accurate estimates of the masses.

- HIP 44248 (10 Uma): $\Delta m$ is 10\% larger and 4 times more accurate. The mass difference between the components is then slightly larger.

- HIP 84140 (Kui 79): A $\Delta m$ about 2 times smaller reduces the mass difference in a significant way. The almost identical small masses obtained are therefore more acceptable for this pair of quasi-similar red dwarfs.

- HIP 93574 (Fin 357): The magnitude difference is significantly larger than the previous estimate, and allows the primary component to recover his logical status of more massive star of the pair. Its mass is actually $14 \%$ larger than the secondary's mass (instead of $22 \%$ smaller before).

- HIP 107354 ( $\kappa$ Peg): The components are this time found to be almost of equal brightness, a fact which increases the mass difference, already fairly large, between the two stars. The respective status of both components is thus not clear, and we should probably exchange them in order to have the primary more massive than the secondary. The spectral types are still needed to check the solution.

\section{The mass luminosity relation}

\subsection{Computation}

The masses and parallaxes derived from the previous study and the Hipparcos magnitudes have been used to determine the relation between the mass and the luminosity of the stars in this sample. This aims firstly to detect anomalous results from the residuals of the fit and secondly to compare this fit to other investigations of the mass-luminosity relation for the early type stars. The absolute magnitudes have been computed by using our parallax solution listed in Tables $7-8$. The individual magnitudes of the components came either directly from the Hipparcos results (when $\Delta m$ 's computation was possible), or from the combination of a ground-based $\Delta m$ with the Hipparcos composite magnitude $H_{\mathrm{p}}$. In any case the relation is,

$H_{1,2}^{\mathrm{abs}}=H_{1,2}+5 \log \pi-10$

where $H_{1}^{\text {abs }}$ and $H_{2}^{\text {abs }}$ are respectively the Hipparcos absolute magnitudes of the primary and secondary components, $\pi$ is the Hipparcos parallax in mas, and $H_{1}, H_{2}$ are the apparent magnitudes, derived by,

$H_{1}=H_{\mathrm{p}}+2.5 \log \left(1+10^{-0.4 \Delta m}\right) ; H_{2}=H_{1}+\Delta m$. 
Table 12. Absolute magnitudes for $52(17+35)$ systems with relative errors on individual masses less than $25 \%$

\begin{tabular}{rrrrrrrrrrrrrrrr}
\hline HIP & $H_{1}^{\text {abs }}$ & $\sigma$ & $H_{2}^{\text {abs }}$ & $\sigma$ & HIP & $H_{1}^{\text {abs }}$ & $\sigma$ & $H_{2}^{\text {abs }}$ & $\sigma$ & HIP & $H_{1}^{\text {abs }}$ & $\sigma$ & $H_{2}^{\text {abs }}$ & $\sigma$ \\
\hline 7918 & 4.60 & 0.03 & 12.05 & 0.50 & 45571 & 3.57 & 0.09 & 3.57 & 0.09 & 83895 & -1.63 & 0.14 & -0.59 & 0.19 \\
8903 & 1.45 & 0.04 & 4.63 & 0.21 & 68682 & 5.34 & 0.04 & 9.64 & 0.49 & 84949 & 2.35 & 0.13 & 2.41 & 0.14 \\
12153 & 3.66 & 0.09 & 3.86 & 0.10 & 71094 & 2.39 & 0.16 & 2.85 & 0.16 & 86722 & 5.88 & 0.06 & 9.84 & 0.49 \\
12623 & 3.75 & 0.10 & 4.05 & 0.12 & 76852 & 1.46 & 0.12 & 1.59 & 0.12 & 95995 & 5.83 & 0.14 & 7.22 & 0.50 \\
20087 & 2.18 & 0.13 & 4.24 & 0.37 & 81126 & -0.24 & 0.14 & 1.37 & 0.28 & 98001 & 4.75 & 0.09 & 5.44 & 0.10 \\
33451 & 3.75 & 0.12 & 4.16 & 0.16 & 82817 & 10.68 & 0.10 & 10.77 & 0.11 & & & & & \\
& & & & & & & & & & & & & & \\
171 & 5.50 & 0.03 & 8.71 & 0.15 & 43671 & 1.53 & 0.14 & 1.54 & 0.14 & 91394 & 3.77 & 0.14 & 3.89 & 0.14 \\
2237 & 4.63 & 0.09 & 4.80 & 0.09 & 44248 & 3.13 & 0.04 & 5.43 & 0.05 & 93574 & 2.75 & 0.12 & 3.13 & 0.15 \\
2762 & 3.96 & 0.05 & 5.25 & 0.07 & 45170 & 5.61 & 0.08 & 6.01 & 0.10 & 94349 & 11.14 & 0.10 & 13.84 & 0.17 \\
7580 & 4.01 & 0.11 & 4.42 & 0.13 & 54204 & 2.52 & 0.11 & 2.62 & 0.11 & 94739 & 9.18 & 0.10 & 9.19 & 0.10 \\
12390 & 2.74 & 0.11 & 2.80 & 0.12 & 75695 & 1.16 & 0.07 & 2.76 & 0.12 & 96683 & 1.20 & 0.12 & 1.50 & 0.14 \\
14328 & -1.03 & 0.14 & 0.53 & 0.18 & 83838 & 2.48 & 0.11 & 2.61 & 0.12 & 104858 & 4.03 & 0.06 & 4.04 & 0.06 \\
14576 & -0.13 & 0.05 & 2.90 & 0.15 & 84140 & 10.93 & 0.08 & 11.16 & 0.10 & 105431 & 4.12 & 0.12 & 4.22 & 0.13 \\
19508 & 4.22 & 0.16 & 4.32 & 0.16 & 86032 & 1.37 & 0.03 & 4.87 & 0.15 & 107354 & 2.22 & 0.09 & 2.32 & 0.09 \\
19719 & 3.14 & 0.11 & 3.29 & 0.11 & 87204 & 4.43 & 0.11 & 4.44 & 0.11 & 108431 & 1.42 & 0.14 & 1.52 & 0.14 \\
24608 & 0.35 & 0.03 & 0.51 & 0.04 & 87655 & 2.97 & 0.13 & 3.17 & 0.14 & 112158 & -0.79 & 0.13 & -2.91 & 0.19 \\
31509 & 3.93 & 0.08 & 4.33 & 0.11 & 87895 & 4.26 & 0.06 & 7.08 & 0.28 & & & & & \\
38052 & 4.94 & 0.10 & 5.04 & 0.11 & 89937 & 4.29 & 0.05 & 6.31 & 0.34 & & & & & \\
\hline
\end{tabular}

The ground-based $\Delta m$ has been expressed in the Hipparcos photometric system when possible (see Sect. 4). The results are listed in Table 12 .

In the following, two sets of data are considered: the first one (Fig. 2) concerns the objects studied in the present paper (excluding the 6 revised stars taken from Paper II), while the second one includes also all the results obtained in our previous work (Figs. 3, 4 and 5). For the first three plots the components with relative error of the mass larger than $25 \%$ have been discarded. This limit is reduced to $13 \%$ in the case of the last plot, Fig. 5 . There are 30 components belonging to 17 systems in the first set and 99 components belonging to 52 systems in the second group, including the non-main sequence objects and the stars lying outside of the main distribution, called "outliers".

The size of each dot in Figs. 2, 3, 4 and 5 is a visual indication of the relative quality of the mass: the bigger dots correspond to the better results $\left(\sigma_{M} / M<10 \%\right)$ and so on to the smaller dots by steps of $5 \%$, up to $25 \%$. The error bars in both $\log \left(M / M_{\odot}\right)$ and absolute magnitude are only represented on the last two plots.

\subsection{The different fits}

We have used a very simple and robust procedure to fit a weighted polynomial model to the data. The obvious outliers have been first excluded from the fit by assigning a null weight to their contribution. Then, two dashed lines parallel to the fitted straight line have been drawn to highlight the main distribution of the scatter, arbitrarily taken at a distance of \pm 0.15 in $\log \left(M / M_{\odot}\right)$. Eventually
Table 13. List of the non-main sequence components excluded from the fit in Figs. 4 and 5

\begin{tabular}{|c|c|c|c|}
\hline HIP & Sp. Type & HIP & Sp. Type \\
\hline $14328 \mathrm{~B}$ & G8III & $91394 \mathrm{~A}+\mathrm{B}$ & F9IV \\
\hline $24608 \mathrm{~A}+\mathrm{B}$ & G1III+G8III & $96683 \mathrm{~A}+\mathrm{B}$ & K0III+K0III \\
\hline $83895 \mathrm{~A}+\mathrm{B}$ & B6III & $107354 \mathrm{~A}+\mathrm{B}$ & F5IV \\
\hline 84949 A & G5IV & $108431 \mathrm{~A}+\mathrm{B}$ & F0IV \\
\hline $86032 \mathrm{~A}+\mathrm{B}$ & A5III & $112158 \mathrm{~A}+\mathrm{B}$ & G2II-III+G8II \\
\hline
\end{tabular}

we could identify additional outliers by this way and iterate the procedure. The rejected systems, shown in Fig. 3, have been discussed on a case by case basis in Sect. 5.1.

The fits are based on a cleaned sample obtained after the removal of every non-main sequence object, identified by their assumed spectral class (SIMBAD database, Hipparcos catalogue, literature), an exception being made for the intermediate class IV-V objects. The rejected systems are listed in Table 13. When the composite spectral class of the pair is the only available information, we assume the components belong to the same class, and thus probably reject more stars than needed. The outliers are naturally also removed and we are left with 75 components of 42 systems when $\sigma_{M} / M<25 \%$ (Fig. 4), or just 32 components of 18 systems if the restriction is pushed to $13 \%$ (Fig. 5 ).

It is well known that a single linear relation cannot fit all mass ranges at a time. The M-L relations for the very high or very low mass stars differ from that concerning the 


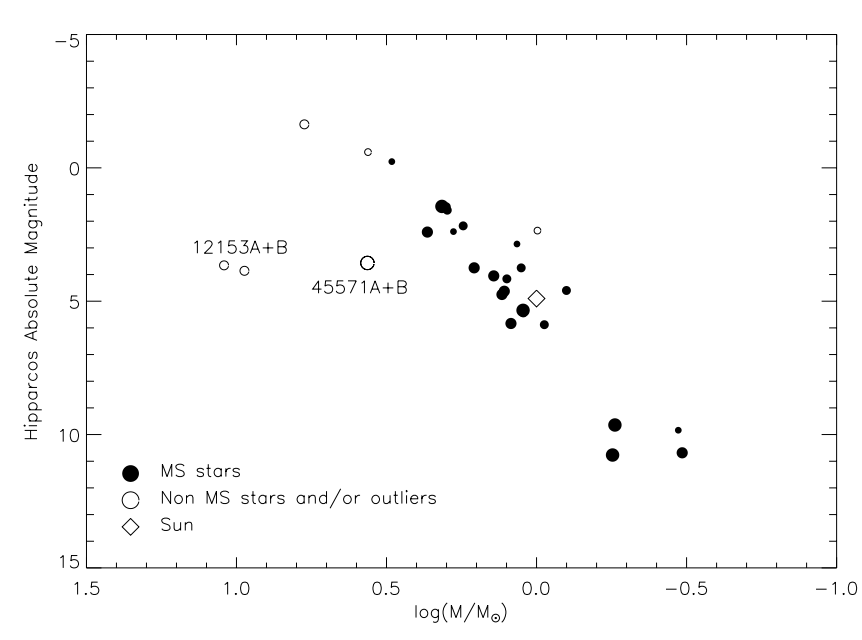

Fig. 2. Mass Luminosity diagram for 30 components belonging to 17 systems studied in this paper, with $\sigma_{M} / M<25 \%$. The dot's size depends on the quality of the mass (see Sect. 6.1). Open circles stand for the components outside the main sequence and for the outliers, the latter being identified by their Hipparcos ID. The diamond represents the position of the Sun, assuming its absolute mag is 4.9. Note: the parameter in ordinate is not the luminosity but the absolute magnitude in the Hipparcos band; this distinction is significant for the stars outside the range $0.6-2.0 M_{\odot}$

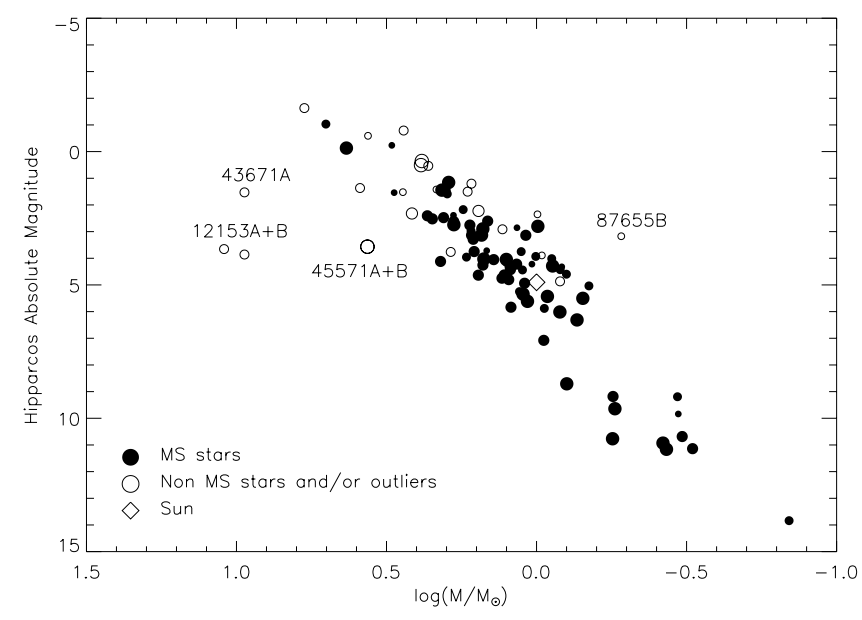

Fig. 3. Same as Fig. 2 for the whole set of binaries: 99 components belonging to 52 systems (compilation of the stars contained in this paper and the previous one)

central part of the diagram (see for example Cester et al. 1983, or Söderhjelm et al. 1997). In the present case, the components with masses larger than $2 M_{\odot}$ or smaller than $0.6 M_{\odot}$ were not taken into account in the fit of the central part. These limits are materialized on the plots by two vertical dotted lines. An additional restriction was applied to the absolute magnitude, namely $H^{\text {abs }}<8$, in order to rule out any ambiguous object. Due to the very small number of objects in the extreme ranges of the diagram, we did not try to fit a relation in these two areas. Thus, the number of stars used in the fit is respectively 54 and 23 in Figs. 4 and 5.

The linear regression was computed in a very classic way, already used by Cester (1983). Briefly, It consists of fitting the data with two different straight lines by minimizing a $\chi^{2}$ function estimated in two orthogonal directions, and by taking the line which intercepts the two previous ones and whose slope is the average of the two slopes. In each case the weights of the data points have been set to $1 / \sigma_{i}^{2}, \sigma_{i}$ representing the standard deviation of the $i^{\text {th }}$ point in $\log (M)$ or in absolute magnitude, depending on the direction considered. The errors of each coefficient of the solution and the correlation coefficient between the two parameters $\log \left(M / M_{\odot}\right)$ and $H^{\text {abs }}$ are also determined.

This gives for the first case (Fig. 4),

$\log \left(M / M_{\odot}\right)=0.625-0.1292 H^{\text {abs }}$

$$
\pm .011 \pm .0024 \text {. }
$$

For this set of early type main sequence stars, the bolometric correction with respect to the Hipparcos absolute magnitude has been determined by Cayrel (1997) and can be rounded to $B C_{\mathrm{Hp}} \approx-0.2$ (or $m_{\mathrm{bol}}=H p-0.2$ ), which is accurate enough in the present context. Using $m_{b_{\odot}}=4.72$, the above fit can be expressed with the luminosity as,

$$
\log \left(L / L_{\odot}\right)=0.033+3.096 \log \left(M / M_{\odot}\right)
$$

$$
\pm .066 \pm .057 \text {. }
$$

In the second case limited to the best solutions (Fig. 5) we get,

$$
\begin{aligned}
\log \left(M / M_{\odot}\right)= & 0.537-0.1074 H^{\mathrm{abs}} \\
& \pm .010 \quad \pm .0020
\end{aligned}
$$

or equivalently for the luminosity,

$$
\begin{gathered}
\log \left(L / L_{\odot}\right)=- \\
-0.032+3.724 \log \left(M / M_{\odot}\right) \\
\pm .079 \quad \pm .070 .
\end{gathered}
$$

The correlation coefficient between the " $X$ " and " $Y$ " variables of the diagram equals 0.62 in the first case (Eqs. (4) and (5)) and 0.70 in the second one (Eqs. (6) and (7)). According to the number of data points in each case, these values show that the linear dependance is highly significant (the probability to produce such correlation values by chance if the variables were independant is less than $0.001)$.

\subsection{Discussion}

We have processed the same data as above with a similar filtering in mass, including this time the non-main sequence objects. It turns out that their influence to the fit is negligible, since each numerical coefficient of Eqs. (4)(7) changes only by about $3 \%$. The restriction in mass is on the other hand very important, even for such a sample with a fairly large scatter. 


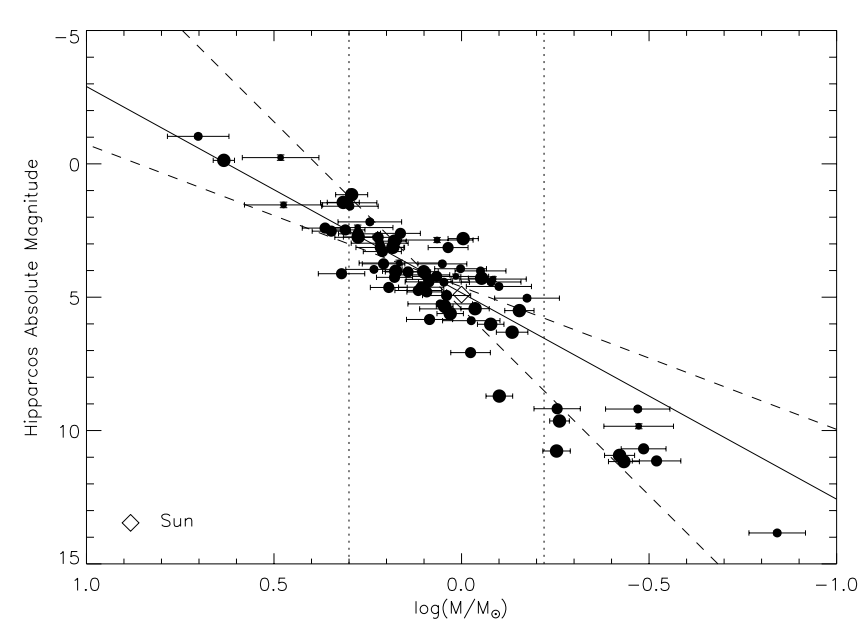

Fig. 4. Mass Luminosity relation fitted over 54 main sequence components with $\sigma_{M} / M<25 \%$ (75 components visible in total). Non-main sequence stars and outliers have been removed. The dot's size depends on the quality of the mass. The two vertical dotted lines indicate the limits of the mass range considered for the fit, represented by the plain straight line. See details in Sect. 6.2. As bolometric corrections were not computed, the scatter's behaviour in the two extreme mass ranges of the diagram is not physically significant. The Sun is at the centre of the diagram (diamond)

The slopes of the mass-luminosity relation are signifcantly different according to the selection threshold. This may indicate that the formal errors in the linear fit are underestimated. One must notice that the procedure to allow for the absence of an independent and perfectly controlled variable in the model fitting, is rather ad-hoc and lacks statistical rigor. Given the precision of the masses, it is however an acceptable approach. The value $K=3.7$ found in the second and more reliable solution agrees well with recent determinations on similar material (Lampens et al. 1997). A more refined solution and thoughtful discussion would not only require an improved knowledge of the masses, but that of the spectral type and luminosity class for every component and was beyond the main scope of this paper.

\section{Conclusion and further works}

This series of papers illustrates meaningfully the scientific interest of combining the high quality astrometry from space with ground based data. On one hand the absolute astrometry from space enables astronomers to track the tiny motion of the photocenter of close binaries with orbital periods less than a couple of decades, while the relative astrometry from the ground carried out over many years is the only efficient source of orbital parameters. The Hipparcos data have been carefully archived and there are still several systems pinpointed by Hipparcos awaiting the

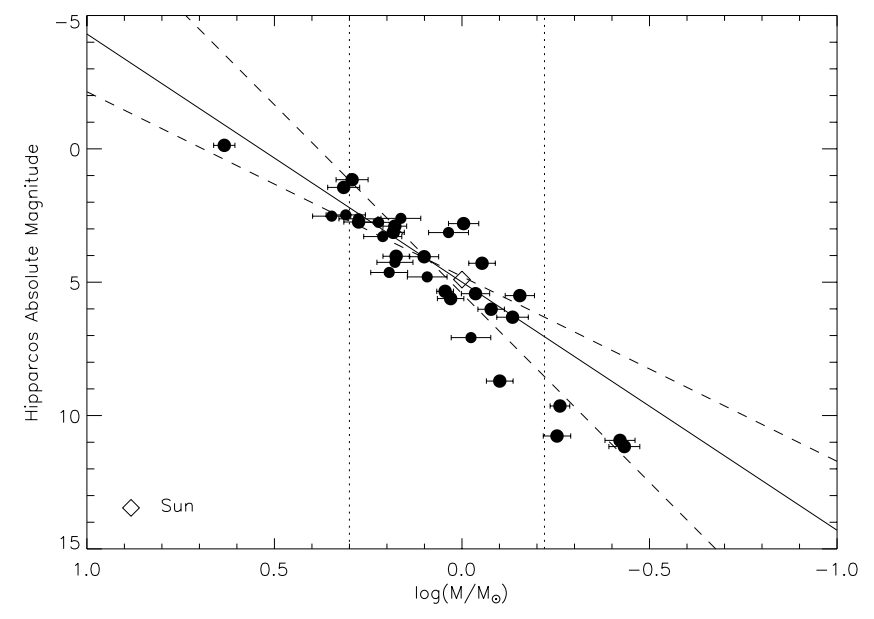

Fig. 5. Same as Fig. 4 (the previous remarks still hold here) with a stronger restriction in mass quality: $\sigma_{M} / M<13 \%$. Over the 32 visible components, 23 are effectively participating to the fit

availability of a better orbit to be checked in view of determining the masses of their components.

Acknowledgements. The data transfers from CERGA to CHARA have been possible through the precious help of J.L. Falin. We have also benefited from the SIMBAD astronomical database, maintained at the CDS, Strasbourg, France. The speckle interferometry program at Georgia State University has been supported by the U.S. National Science Foundation, most recently through Grant No. AST94-16994, by the office of the Dean of the College of Arts and Sciences, and by the Research Program Enhancement program administrated by the office of the Vice President for Research and Sponsored Programs at GSU.

\section{References}

Africano J.L., et al., 1978, AJ 83, 1100

Baize P., 1950, J. Obs. 33, 1

Baize P., 1989, A\&AS 81, 415

Baize P., 1991, A\&AS 87, 49

Barlow D.J., Scarfe C.D., 1991, AJ 102, 2098

Balega I.I., Balega Y.Y., 1988, Sov. Astron. Lett. 14, 393

Blazit A., 1977, ApJ 214, L79

Cayrel R., et al., 1997, Proceedings of the ESA Symposium "Hipparcos - Venice'97", ESA SP-402, 433

Cester B., Ferluga S., Boehm C., 1983, Astrophys. Space Sci. 96, 125

Colacevich A., 1935, PASP 47, 284

Colacevich A., 1941, Oss. e. Mem. Arcetri 59, 15

Docobo J.A., Costa J.M., 1984, Circ. Inf., No. 92

Duquennoy A., Tokovinin A.A., 1996, A\&A 314, 846

Duquennoy A., Mayor M., 1991, A\&A 248, 485

ESA, 1997, The Hipparcos and Tycho Catalogues, SP-1200

Finsen W.S., 1963, Republic Obs. Circ. 7, 39

Finsen W.S., 1964, J. Obs. 47, 98

Griffin R.F., 1977, The Obs. 97, 15 
Griffin R.F., 1990, The Obs. 110, 177

Hartkopf W.I., et al., 1989, AJ 98, 1014

Hartkopf W.I., et al., 1992, AJ 103, 1976

Hartkopf W.I., et al., 1996, AJ 111, 370

Heintz W.D., 1984a, Circ. Inf., No. 93

Heintz W.D., 1984b, AJ 89, 1063

Heintz W.D., 1990, A\&AS 82, 65

Heintz W.D., 1991, A\&AS 90, 311

Heintz W.D., 1994, AJ 108, 2388

Heintz W.D., 1997, ApJS 111, 335

Henry T.J., et al., 1992, AJ 103, 1369

Henry T.J., et al., 1993, AJ 106, 773

Jenkins L.F., 1963, General Catalog of Trigonometric Parallaxes

Kamper K.W., 1987, AJ 93, 683

Lampens P., et al., 1997, Proceedings of the ESA Symposium "Hipparcos - Venice'97", ESA SP-402, 421

Lindegren L., et al., 1997, A\&A 323, L53-56

Lippincott S.L., et al., 1983, PASP 95, 271

McAlister H.A., 1978, ApJ 223, 526

McAlister H.A., 1976, PASP 88, 317

Martin C., Mignard F., Frœschlé M., 1997, A\&AS 122, 571-580

Martin C., Mignard F., 1998, A\&A 330, 585

Mason B.D., 1997a, ApJ 114, 808
Mason B.D., McAlister H.A., Hartkopf W.I., 1997b, AJ 114, 1607

Mazeh T., et al., 1992, AJ 401, 265

Mignard F., et al, 1992, A\&A 258, 165

Mignard F., et al., 1996, A\&A 304, 94

Olević D., Jovanović P., 1997, Bull. Astron. Belgrade 155, 103

Pan X.P., et al., 1990, ApJ 356, 641

Scarfe C.D., et al., 1994, AJ 107, 1529

Söderhjelm, et al., 1997, Proceedings of the ESA Symposium "Hipparcos - Venice'97", ESA SP-402, 251

Tokovinin A.A., 1986, Soviet. Astron. Lett. 12, 480

Van den Bos W.H., 1956, Union Obs. Circ. 6, 279

Van Hamme W.V., et al., 1994, AJ 107, 1521

Voronov. N., 1934, Tashkent Obs. Circ, 27

Wasson R., et al., 1994, AJ 107, 1514

Worley C.E., Heintz W.D., 1983, Fourth Catalog of Orbits of Visual Binary Stars, Publ. U.S. Naval Obs.

Worley C.E., Douglass G.G., 1996, The Washington Visual Double Star Catalog, Publ. U.S. Naval Obs.

Zombeck M.V., 1990, Handbook of Space Astronomy \& Astrophysics, Cambridge U.P.

Zulević D.J., 1992, Bull. Astron. Obs. Belgrade 146, 61

Zulević D.J., 1993, Circ. Inf., No. 119 